\title{
Vortex-line liquid phases: longitudinal superconductivity in the lattice London model
}

\author{
T.J. Hagenaars ${ }^{1}$, E.H. Brandt ${ }^{2}$, R.E. Hetzel ${ }^{1, *}$, W. Hanke ${ }^{1}$, M. Leghissa ${ }^{3, * *}$, and G. Saemann-Ischenko ${ }^{3}$ \\ ${ }^{1}$ Institut für Theoretische Physik, Universität Würzburg \\ Am Hubland, 97074 Würzburg, Germany \\ ${ }^{2}$ Max-Planck-Institut für Metallforschung, Institut für Physik \\ Postfach 800665, 70506 Stuttgart, Germany \\ ${ }^{3}$ Physikalisches Institut III, Universität Erlangen-Nürnberg \\ Erwin-Rommel-Strasse 1, 91058 Erlangen, Germany
}

\begin{abstract}
We study the vortex-line lattice and liquid phases of a clean type-II superconductor by means of Monte Carlo simulations of the lattice London model. Motivated by a recent controversy regarding the presence, within this model, of a vortex-liquid regime with longitudinal superconducting coherence over long length scales, we directly compare two different ways to calculate the longitudinal coherence. For an isotropic superconductor, we interpret our results in terms of a temperature regime within the liquid phase in which longitudinal superconducting coherence extends over length scales larger than the system thickness studied. We note that this regime disappears in the moderately anisotropic case due to a proliferation, close to the flux-line lattice melting temperature, of vortex loops between the layers.
\end{abstract}

\section{INTRODUCTION}

The effect of strong thermal fluctuations on the flux lines is an important aspect of the physics of high- $T_{c}$ superconductors in the mixed state. It is believed that these fluctuations melt the ground-state flux-line lattice into a vortex-line liquid below $T_{c}$ [1.22].

In Nelson's analogy of vortex lines with world lines of $2 \mathrm{D}$ bosons [3], the absence of superfluidity of the bosons corresponds to the presence of superconducting coherence along the field direction in the vortex-line system 听, . The liquid phase without longitudinal superconductivity (sometimes referred to as an "entangled vortex liquid") corresponds to the 2D boson superfluid state. Similarly, the vortex-line liquid with longitudinal superconductivity ("disentangled vortex liquid") corresponds to the normal liquid state of the $2 \mathrm{D}$ bosons. The quantity corresponding to the inverse 2D-boson temperature in the vortex-line system is the system size $L_{z}$ along the field direction. Therefore, for a small enough $L_{z}$, Nelson's analogy predicts longitudinal superconducting coherence in the vortex liquid. In Ref. [5] it has been argued that the disentangled vortex liquid exists as a true thermodynamic phase $\left(L_{z} \rightarrow \infty\right)$, at least for the case where the distance between the vortex lines is (much) smaller than the magnetic penetration depth $\lambda_{1}$. The scenario in which the longitudinal superconductivity is destroyed already within the vortex-lattice phase, leading to a "supersolid" phase, has been considered in [6].

There exists a substantial recent literature that deals with $\mathrm{MC}$ simulations of the vortex-line phases, using various model approximations [4, 月, 16]. In two recent papers, in which the vortex-line system was studied within lattice London models [4, 14, opposite conclusions have been reached regarding the presence of longitudinal supercon- ductivity within the vortex-liquid phase for systems with $L_{z} \sim 15-30$ lattice constants. Although in both works cited, the longitudinal coherence was argued to exist only over a finite length scale (and therefore no true disentangled vortex-liquid phase was claimed to exist), a difference of two orders of magnitude in the results for this length scale translates into opposite predictions for the experimentally observable behavior in typical samples studied in recent flux-transformer experiments, for temperatures above but close to melting. One of the motivations of our work has been to clarify the reason(s) for the above disagreement. On the one hand, Chen and Teitel [4], who perform the Monte Carlo simulation at constant magnetic induction $B$, found that longitudinal superconductivity persists far into the vortex liquid for $L_{z}=30$ lattice constants. These authors argue that the length scale beyond which the vortex lines become entangled, corresponds to approximately 410 lattice constants. For YBCO this length scale for longitudinal coherence was estimated in [4] to be much larger than the thickness of the samples studied in recent flux-transformer experiments [29,30]. As a result, their findings suggest the existence of a temperature regime in these experiments, in which the vortex lattice has melted but in which the flux motion is still maximally $z$-correlated, in agreement with the results obtained for twinned YBCO samples [29]. On the other hand Carneiro 14 found evidence for the disappearance of the vortex-liquid regime with disentangled lines already between $L_{z}=6$ and $L_{z}=12$. These results were argued to agree with later experiments on untwinned YBCO samples, in which the flux-line lattice melting and a loss of maximally $z$-correlated vortex motion was found to coincide.

In Carneiro's calculations the longitudinal response is calculated in a different way, discussed in more detail below, in which fluctuations in the net vorticities (in 
two directions) are allowed. In a recent Comment [17, Chen and Teitel have criticized Carneiro's calculational scheme. They suggested that it effectively measures the transverse instead of the longitudinal response. Below, we will present results of MC simulations of the lattice London model. We present a direct comparison of the two different calculational schemes, and will point out that the method of Ref. 14] has some serious problems.

From our results we conclude that the isotropic lattice London model predicts a temperature regime in which longitudinal coherence over long length scales exists within the vortex-liquid phase. We also present results for a moderately anisotropic system, in which the temperature at which longitudinal coherence is lost, is roughly equal to the melting temperature.

The outline of this paper is as follows. In section II we introduce the model and the Monte Carlo method(s). In section III we present our results. We summarize our findings in section IV.

\section{LATTICE LONDON MODEL AND MC ALGORITHMS}

The first MC study of the lattice London model was performed by Carneiro, Cavalcanti and Gartner [18]. The model was explored further by Carneiro for a superconductor with free surfaces [19]. The lattice London model is formulated directly in terms of the vortex degrees of freedom. The vortex lines are modeled as consisting of elements of unit length based at dual lattice sites of a cubic lattice. At every dual lattice site we define three integers $q_{\mu}(\boldsymbol{R})=0, \pm 1, \pm 2, \ldots$, the vorticities in the directions $\mu=x, y, z$. When a vorticity $q_{\mu}\left(\boldsymbol{R}_{i}\right)$ is nonzero, its magnitude gives the number of flux quanta carried by the associated vortex-line element. The $q_{\mu}\left(\boldsymbol{R}_{i}\right)$ are subject to the continuity constraint

$$
\sum_{\boldsymbol{e}_{\mu}}\left[q_{\mu}\left(\boldsymbol{R}_{i}\right)-q_{\mu}\left(\boldsymbol{R}_{i}-\boldsymbol{e}_{\mu}\right)\right]=0
$$

that ensures that the vortex-line elements form either closed loops or lines that end at the boundaries. Here $\boldsymbol{R}_{i}-\boldsymbol{e}_{\mu}$ runs over nearest neighbor sites of $\boldsymbol{R}_{i}$. At constant $B$, the Hamiltonian of the isotropic lattice London model, expressed in terms of the vorticities, is given by:

$$
\mathcal{H}=4 \pi^{2} J \sum_{i, j, \mu} q_{\mu}\left(\boldsymbol{R}_{i}\right) q_{\mu}\left(\boldsymbol{R}_{j}\right) g_{\mu}\left(\boldsymbol{R}_{i}-\boldsymbol{R}_{j}\right)
$$

where $J=\Phi_{0}^{2} d /\left(32 \pi^{3} \lambda_{1}^{2}\right)$ and $g_{\mu}(\boldsymbol{R})$ is the London interaction with Fourier components

$$
\begin{gathered}
g_{x, y}(\boldsymbol{k})=\frac{R}{\kappa_{1}^{2}+\kappa_{2}^{2}+R\left(\kappa_{3}^{2}+\left(d / \lambda_{1}\right)^{2}\right)} \\
g_{z}(\boldsymbol{k})=\frac{\kappa^{2}+R\left(d / \lambda_{1}\right)^{2}}{\left(\kappa^{2}+\left(d / \lambda_{1}\right)^{2}\right)\left(\kappa_{1}^{2}+\kappa_{2}^{2}+R\left(\kappa_{3}^{2}+\left(d / \lambda_{1}\right)^{2}\right)\right)}
\end{gathered}
$$

where $\kappa_{\mu}^{2}=2-2 \cos k_{\mu}\left(k_{\mu}=2 \pi n_{\mu} / L_{\mu}, n_{\mu}=\right.$ $\left.0,1, \ldots, L_{\mu}-1\right)$ and $\kappa^{2}=\sum_{\mu} \kappa_{\mu}^{2}$. Here we assumed an $L_{x} \times L_{y} \times L_{z}$ lattice with periodic boundary conditions. $R=\lambda_{1}^{2} / \lambda_{3}^{2}=1 / \Gamma^{2}$ is the anisotropy parameter, with $\lambda_{1}$ and $\lambda_{3}$ the magnetic penetration depths along the $x-y$ plane and the $z$ axis, respectively, and $d$ the lattice constant. The Hamiltonian (2) can be either derived from the discrete version of the London free energy [18,19. or by a duality transformation of the Hamiltonian of a Villain-type lattice superconductor 20].

Monte Carlo sampling of the phase space for the variables $q_{\mu}(\boldsymbol{R})$ at constant $B$ is performed as follows. The initial configuration is prepared to contain the number of vortex lines we want to study, depending on the value chosen for the magnetic induction $B$. A Monte Carlo update step consists of adding at a given site a closed $d \times d$ square loop of unit vorticity with an orientation chosen randomly from the six possible ones. The addition of a closed loop preserves the constraint (11). The standard Metropolis algorithm is employed to accept or reject the new configuration. Obviously, when only closed loops are added, the magnetic induction $B$ with components

$$
B_{\mu}=\frac{\Phi_{0}}{d^{2} V} \sum_{j}\left\langle q_{\mu}\left(\boldsymbol{R}_{j}\right)\right\rangle
$$

( $\left.V=L_{x} L_{y} L_{z}\right)$ will be constant throughout the simulation.

To probe superconducting coherence one can consider the helicity moduli introduced by Chen and Teitel 21, 4, $\Upsilon_{\mu}=\lim _{k_{\nu} \rightarrow 0} \Upsilon_{\mu}\left(k_{\nu}\right)$. Here $\Upsilon_{\mu}\left(k_{\nu}\right)$ is the linear response coefficient between a perturbation $\delta A_{\mu}^{e x t}\left(k_{\nu}\right) \boldsymbol{e}_{\mu}$ of the external vector potential and the induced supercurrent

$$
j_{\mu}\left(k_{\nu}\right)=-\Upsilon_{\mu}\left(k_{\nu}\right) \delta A_{\mu}^{e x t}\left(k_{\nu}\right),
$$

where $\boldsymbol{k} \cdot \boldsymbol{A}^{\text {ext }}=0$ (London gauge). For our lattice London model these moduli are given by

$$
\begin{array}{r}
\Upsilon_{\mu}\left(k_{\nu}\right)=\left.\frac{J \lambda_{1}^{2} \kappa^{2}}{1+\lambda_{1}^{2} \kappa^{2}}\right|_{k_{\mu}=k_{\sigma}=0} \times \gamma_{\mu}\left(k_{\nu}\right), \\
\gamma_{\mu}\left(k_{\nu}\right) \equiv 1-\frac{4 \pi^{2} J \lambda_{1}^{2}}{V k_{B} T} \frac{\left\langle q_{\sigma}\left(k_{\nu}\right) q_{\sigma}\left(-k_{\nu}\right)\right\rangle}{1+\left(1+\delta_{\mu, z}(1 / R-1)\right) \lambda_{1}^{2} \kappa_{\nu}^{2}}
\end{array}
$$

where $(\mu, \nu, \sigma)$ is a cyclic permutation of $(x, y, z)$, $q_{\sigma}\left(k_{\nu}\right) \equiv q_{\sigma}\left(k_{\nu}, k_{\mu}=k_{\sigma}=0\right)$, and $\langle.$.$\rangle denotes a$ thermal average. We will focus on the quantity $\gamma_{z} \equiv$ $\lim _{k_{x} \rightarrow 0} \gamma_{z}\left(k_{x}\right)$. We note that for a uniaxial superconductor with $B$ parallel to the $z$ axis, one has $\gamma_{z}=$ $\lim _{k_{y} \rightarrow 0} \gamma_{z}\left(k_{y}\right)$ from symmetry, where $\gamma_{z}\left(k_{y}\right)$ is defined by the expression (6) with the indices $x$ and $y$ interchanged. If $\gamma_{z}=1$, one has a perfect screening of the corresponding perturbation of the vector potential, and thus longitudinal superconducting coherence. A sharp jump in $\gamma_{z}$ from unity is interpreted as loss of longitudinal coherence. In Ref. [21] it has been shown that $\gamma_{z}$ will be non-zero as long as the vortex-line system retains a finite shear modulus at finite wave vector. Physically, 
this may be understood by the finding of Ref. [22] that helical instabilities of the vortex lines driven by a longitudinal current, can be stabilized either by pinning or by a finite shear modulus.

In the MC calculations, $\gamma_{\mu}$ is estimated from the quantity $\gamma_{z}\left(k_{\nu}\right)$ at small $k_{\nu}$. In Ref. [- 1 . this was done by extrapolation of the results for $\left\langle q_{\sigma}\left(k_{\nu}\right) q_{\sigma}\left(-k_{\nu}\right)\right\rangle$ to $k_{\nu}=0$ using a fit to a polynomial in $\kappa_{\nu}^{2}$. It turns out that such a fit does not work for higher values of $\lambda$ and/or $\Gamma$ than considered in [1]. In Ref. 15] this has been demonstrated for $\lambda=12, \Gamma=1$. However, this problem disappears if one examines the full ratio that defines $1-\gamma_{\mu}\left(k_{\nu}\right)$ (see Eq. (6)). This quantity has only a weak dependence on $k_{\nu}$ [23, so that one already obtains a reliable estimate for $1-\gamma_{\nu}$ from the data point for the smallest nonzero wave vector, $k_{\nu}=2 \pi / L_{\nu}$. This direct way of estimating the response was employed before in Ref. [16]. We illustrate it with an example in Figs. 10 and 11. In particular, $1-\gamma_{z}\left(k_{x}\right)$ is almost independent of $k_{x}$ (when $B$ is along $z$ ). Therefore, it is possible to obtain meaningful results for $\gamma_{z}$ already for systems with $L_{x}$ equal to only a few times the average distance between the vortex lines.

In Ref. [14], a different set up was introduced for measuring longitudinal superconducting coherence. This original set up starts from the observation that at a free boundary parallel to the $y$ - $z$ plane, superconducting coherence in the $z$ direction corresponds to Meissner shielding of an infinitesimal uniform applied field in the $y$ direction 22. For a lattice London model in the form of a slab, with $\mathrm{fbc}$ in the $x$ direction and pbc in the other directions, and in a constant applied magnetic field, this shielding is measured by the transverse magnetic permeability $\mu_{y}$.

$$
\mu_{y}=\left.\frac{\partial B_{y}}{\partial H_{y}}\right|_{H_{y}=0}=\frac{V d^{3}}{4 \pi k_{B} T}\left\langle b_{y}^{2}\right\rangle,
$$

where $\langle.$.$\rangle is now a thermal average weighted by the$ Hamiltonian

$$
\tilde{\mathcal{H}}=\mathcal{H}-\frac{V d^{3}}{4 \pi} \Phi_{0} / d^{2} \sum_{\mu} f_{\mu} b_{\mu}
$$

Here $f_{\mu}=H_{\mu} d^{2} / \Phi_{0}$ is the dimensionless applied magnetic field in units of the elementary flux quantum $\Phi_{0}$ per plaquette area. In (8) $f_{\mu}$ multiplies the microscopic magnetic field $b_{\mu}$ :

$$
b_{\mu}=\frac{\Phi_{0}}{d^{2} V} \sum_{i} q_{\mu}\left(\boldsymbol{R}_{i}\right) .
$$

When $\mu_{y}$ is zero, the external field $H_{y}$ is shielded by supercurrents running parallel to $\boldsymbol{e}_{z}$ and thus the system is a longitudinal superconductor. When $\mu_{y}$ is nonzero, this is interpreted as a loss of longitudinal superconductivity. Note that $\mu_{y}=1-\gamma_{z}\left(k_{x}=0\right)$, which is identically zero in the case of periodic boundaries and constant $B$. For a slab placed in a constant applied magnetic field however, the vorticity fluctuations measured by the thermal average in the right hand side of Eq. (7) can be nonzero by virtue of the free boundaries. At such boundaries the elementary vorticity fluctuations satisfying the constraint (11) are elementary loops with one side missing (the side outside the sample), so-called incomplete loops.

The MC method at constant applied field, introduced by Carneiro in Ref. [14], uses the vortex-vortex interaction $g(\boldsymbol{R})$ for a system with periodic boundary conditions in all directions, but updates at sites in the planes $x=1$ and $x=L_{x}$ as if these planes were (adjacent to) free boundaries, allowing the addition of closed elementary loops parallel to the $y-z$ plane and of incomplete loops parallel to the $x-y$ and $x-z$ planes. The periodic boundary conditions are imposed also in the $x$ direction (through the form of $g(\boldsymbol{R})$ ) to diminish finite size effects. Although $x=1$ and $x=L_{x}$ are therefore no genuine free boundaries, Carneiro has claimed that the addition of incomplete loops incorporates the essence needed for the calculation of $\mu_{y}$. It is however not a priori evident whether or not this treatment of the boundaries leads to meaningful results for $\mu_{y}$. In fact, we think that it does not, as we will argue from a consistency check presented in paragraph III.A.

In Carneiro's method, the allowed incomplete loops do not lead to net vorticity fluctuations in the $x$ direction. We note that this allows us to simultaneously extract $\gamma_{z}$ from a simulation in which we calculate $\mu_{y}$, namely from the fluctuations of $q_{x}$ at $k_{x}=k_{z}=0$ and the smallest nonzero $k_{y}$. As a result, we are able to compare the results of the two methods in one and the same simulation.

\section{RESULTS}

We have considered vortex-line systems corresponding to $B=1 / 8, B=1 / 15$ and $B=1 / 24$ ( $B$ along $z$ and measured in units of $\left.\Phi_{0} / d^{2}\right)$. The corresponding groundstate vortex lattices are shown in Fig. 1. (a)

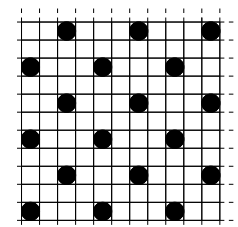

(b)

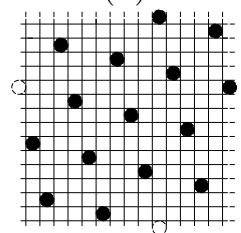

(c)

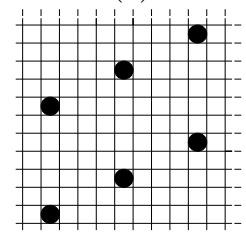

FIG. 1. Ground state vortex lattices, projected onto the $x-y$ plane, for different values of the dimensionless magnetic field $f$ : (a) $B=1 / 8$, (b) $B=1 / 15$, (c) $B=1 / 24$.

For each temperature we start in the ground-state configuration. Runs consist of $16384 \mathrm{MC}$ sweeps through the lattice. Of these the first 8192 sweeps are discarded for equilibration, and the second 8192 are used to compute the thermal averages. 


\section{A. Isotropic case.}

We first present results obtained employing Carneiro's method, for different values of the magnetic field $f_{x}=$ $f_{y}=0, f_{z}=f$. For $B=1 / 8$ and $B=1 / 24$ we chose the commensurate lattice size $L_{x}=L_{y}=L_{z}=12$ and for $B=1 / 15$ the sizes $L_{x}=L_{y}=15, L_{z}=6,15$. In Fig. 2 we show the vortex-lattice correlations for vortex elements $q_{z}\left(\boldsymbol{R}_{i}\right)$ in the $z$-direction:

$$
P(\boldsymbol{R})=\frac{\sum_{j}\left\langle q_{z}\left(\boldsymbol{R}_{j}+\boldsymbol{R}\right) q_{z}\left(\boldsymbol{R}_{j}\right)\right\rangle}{\sum_{j}\left\langle q_{z}^{2}\left(\boldsymbol{R}_{j}\right)\right\rangle}
$$

as a function of temperature, for $B=1 / 8, \lambda_{1}=12$; they are in quantitative agreement with the corresponding results reported in Refs. 114 and 15]. We measure the temperature in units of the coupling $J / k_{B}$. The melting of the flux-line lattice is reflected in the decay of the corresponding correlations. From the curves in Fig. 2 we may estimate the melting temperature to be $T_{m}=2.7 \pm 0.1$. This estimate is actually an upper bound of the true melting temperature. As has been noted in Refs. [4, 16], the discrete mesh introduces an artificial pinning potential for the vortex lines that tends to increase the apparent melting temperature. This effect is reduced when considering smaller vortex densities, that correspond to a finer effective mesh. In [16] the depinning from the mesh was argued to occur below the (true) melting temperature for $B$ lower than approximately $B_{c}=1 / 32$.

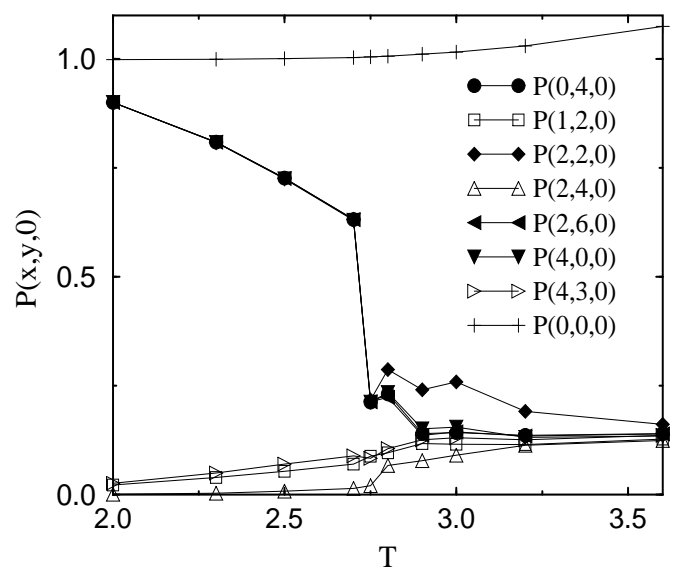

FIG. 2. Vortex-vortex spatial correlations $P(\boldsymbol{R})$ for $B=$ $1 / 8, \lambda_{1}=12, L_{x}=L_{y}=L_{z}=12$ as a function of temperature. Temperature is measured in units of $J / k_{B}$. The filled symbols represent correlation functions that measure the translational order corresponding to the ground-state configuration depicted in Fig. 1(a).

As an illustration of the liquid phase, in Fig. 3 a snapshot of the vortex configuration for the $B=1 / 15$, $\lambda_{1}=15$, and $T=2.4=1.2 T_{m}$ is shown. In this snapshot we observe a few (vortex-line segments built up of) incomplete elementary loops that were generated in the planes $x=1$ and $x=L_{x}$.

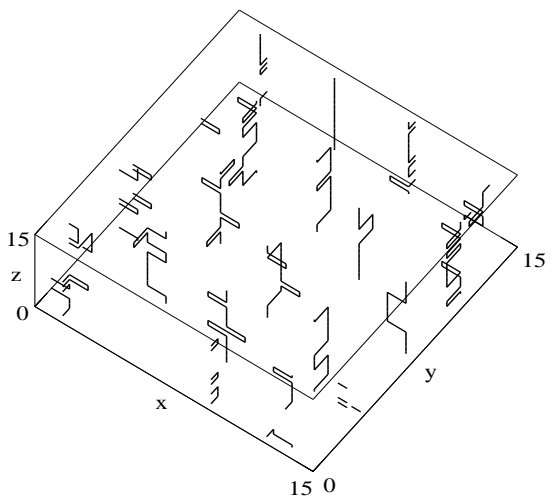

FIG. 3. Snapshot of the vortex-line configuration in the liquid phase for $B=1 / 15, \lambda_{1}=15, L_{x}=L_{y}=L_{z}=15$, $T=2.4$. The perspective is from above, the upper and lower $x-y$ planes are enclosed by thin lines, and the fat lines indicate the vortex lines. Note that periodic boundary conditions are imposed in all directions, and that at some places different parts of one and the same vortex line connect through these boundaries. Due to the introduction of incomplete elementary loop fluctuations in the planes $x=1$ and $x=15$, the microscopic field is allowed to fluctuate (see text).

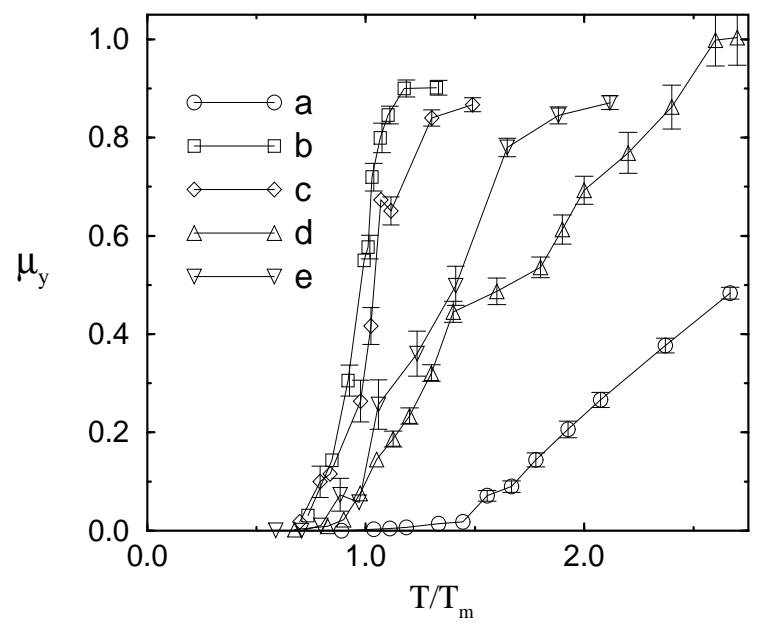

FIG. 4. The transverse response $\mu_{y}$ as a function of temperature, for the parameters (a) $B=1 / 15, \lambda_{1}=5, L_{x}=$ $L_{y}=15, L_{z}=6$, (b) $B=1 / 8, \lambda_{1}=12, L_{x}=L_{y}=L_{z}=12$, (c) $B=1 / 15, \lambda_{1}=15, L=15$, (d) $B=1 / 15, \lambda_{1}=5$, $L_{x}=L_{y}=L_{z}=15$, and (e) $B=1 / 24, \lambda_{1}=12, L=12$. Sample error bars are shown. For all curves the temperature is normalized to the melting temperature $T_{m}$. These melting temperatures are $T=1.35 \pm 0.1$ (a) and $T=2.7 \pm 0.1(\mathrm{~b})$, $T=2.15 \pm 0.1$ (c), $T=2.0 \pm 0.1$ (d), $T=1.7 \pm 0.1$ (e), and have been estimated from the decay of the vortex-lattice correlations as shown in Figs. 2 and 3 [25]. 
In Fig. 4, curve (b) shows $\mu_{y}$ as a function of temperature for $B=1 / 8, \lambda_{1}=12$. As in Refs. [14, 15], $\mu_{y}$ is non-zero above the melting temperature. Curve (d) corresponds to $B=1 / 15, \lambda=5$, the case studied at constant $B$ in Ref. [1]. Here $\mu_{y}$ is still small at the melting temperature, but rises fast for slightly higher temperatures. For $L_{z}=6$ (curve (a)) we find that $\mu_{y}$ stays close to zero in a substantial temperature regime above $T_{m}$. As has been noted before in Ref. [15], this shows that $\mu_{y}$ does not correspond to the transverse response $1-\gamma_{x}$, as was suggested in Ref. [17], because the latter response is resistive in the liquid phase. For further comparison, we have included results for $B=1 / 15, \lambda=15$ (c) and $B=1 / 24, \lambda=12(\mathrm{e})$. For all cases (b)-(e), with $L_{z}=12$ or $L_{z}=15, \mu_{y}$ starts to rise at around the melting temperature. This is in sharp contrast with the results reported for $\gamma_{z}$ in Refs. [4, 16].

We have also extracted $\gamma_{z}$ from these same simulations, in the way explained in Sec II. In Fig. 5 we compare $\mu_{y}$ (triangles) and $1-\gamma_{z}$ (circles) for $B=1 / 15, \lambda_{1}=$ $5, L_{z}=15$, the same parameters that were considered in Ref. [1]. Up to $T=2.5 T_{m}$, these results for $\gamma_{z}$ are within error bars identical to those for $\gamma_{z}$ calculated using the algorithm of Ref. [4] (shown as squares). We find that $1-\gamma_{z}$ rises from close to zero to close to one at a temperature approximately twice as large as $T_{m}$. Similarly, for all other curves in Fig. 4 with exception of curve (b) $(f=1 / 8)$, we find that $\gamma_{z}$ is close to one up to the highest temperature values shown. Therefore, the temperature at which $\gamma_{z}$ drops from unity lies well above the melting temperature, in agreement with the results ob- tained in Refs. 四 and [16]. For $B=1 / 8$ (curve (b) in Fig. $4, \lambda=12$ ) the temperature region between the drop of $P(\boldsymbol{R})$ and the drop of $\gamma_{z}$ is smaller. This is probably caused by the pinning artefact due to the discrete mesh, that tends to increase the apparent melting temperature more for $B=1 / 8$ than for the lower vortex densities studied here 24].

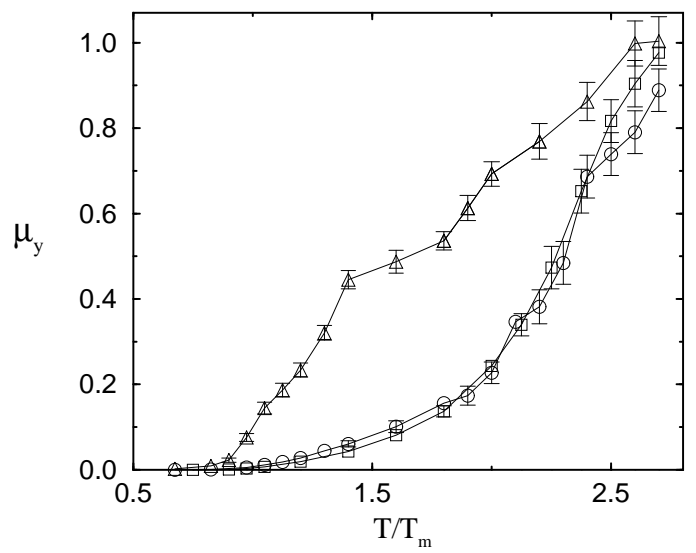

FIG. 5. Comparison of the responses $\mu_{y}$ (triangles) and $1-\gamma_{z}$ (circles) for $B=1 / 15, \lambda_{1}=5, L_{x}=L_{y}=L_{z}=15$ as a function of temperature. Temperature is measured in units of $J / k_{B}$. The data points indicated with squares are results for $1-\gamma_{z}$ from an independent simulation using the MC method with constant $B$. Sample error bars for $1-\gamma_{z}$ refer to the error in the data point for the smallest nonzero $k_{\nu}$ (see text).

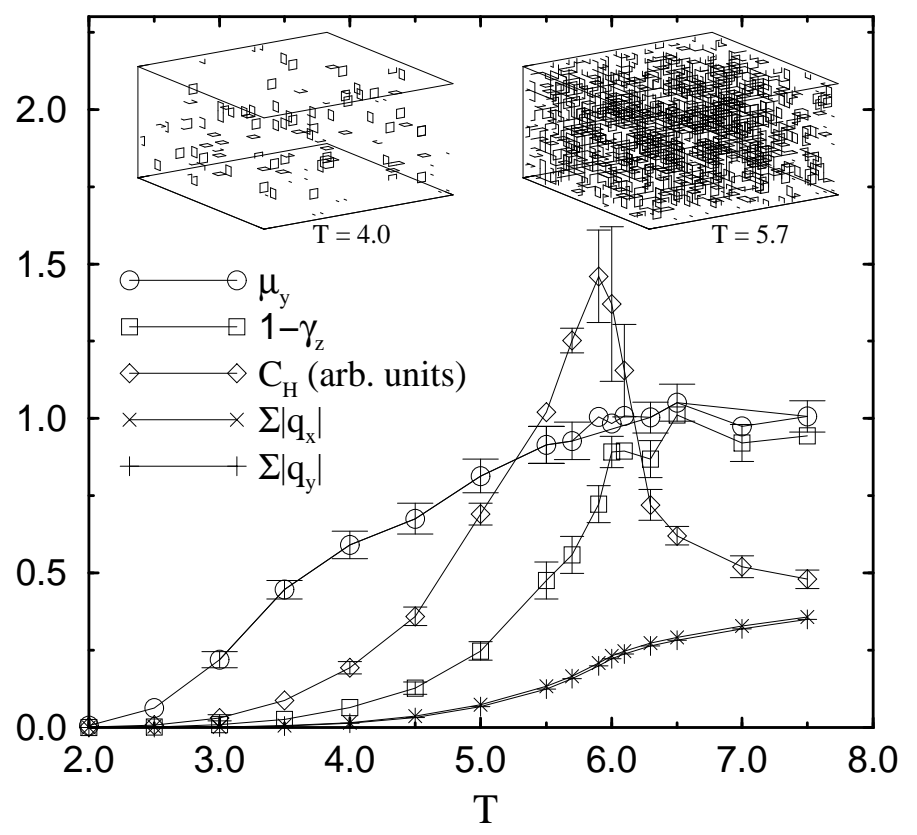

FIG. 6. Responses $\mu_{y}, 1-\gamma_{z}$, specific heat $C_{H}$, and absolute vorticities for $B=0, \lambda_{1}=12, L_{x}=L_{y}=24, L_{z}=12$. Sample error bars are shown for $\mu_{y}, 1-\gamma_{z}$, and $C_{H}$. The insets show snapshots of the vorticity configurations at $T=4.0$ and $T=5.7$. 


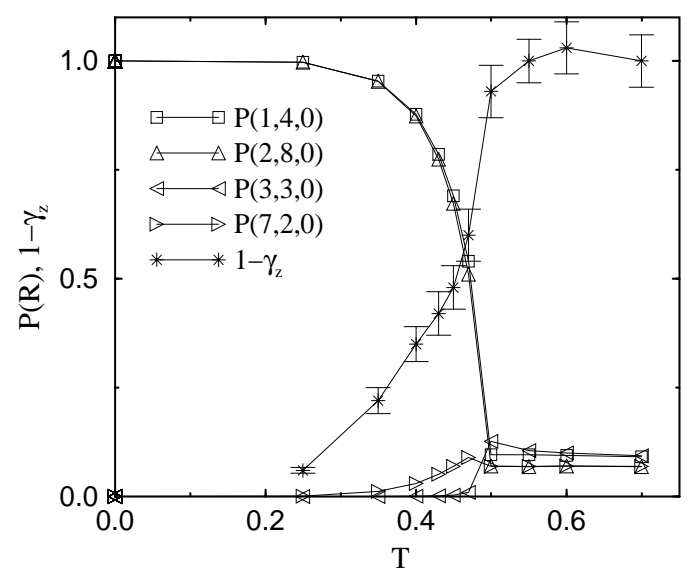

FIG. 7. Vortex-vortex spatial correlations $P(\boldsymbol{R})$ for $R=$ 0.04, $B=1 / 15, \lambda_{1}=5, L_{x}=L_{y}=30, L_{z}=10$ as a function of temperature, together with $1-\gamma_{z}$.

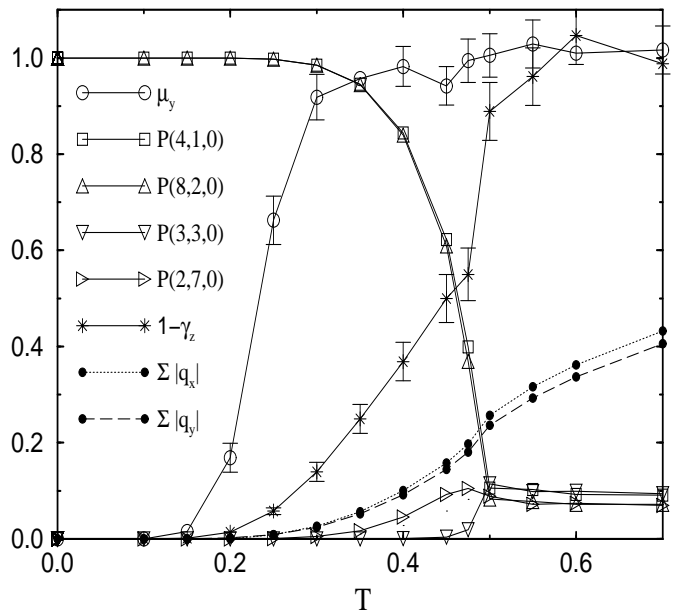

FIG. 8. Vortex-vortex spatial correlations $P(\boldsymbol{R})$ for $R=$ $0.04, B=1 / 15, \lambda_{1}=5, L_{x}=L_{y}=L_{z}=15$ as a function of temperature, together with $\mu_{y}, 1-\gamma_{z}$, and the absolute vorticities in $x$ and $y$ direction. Sample error bars are shown for $\mu_{y}$ and $1-\gamma_{z}$.

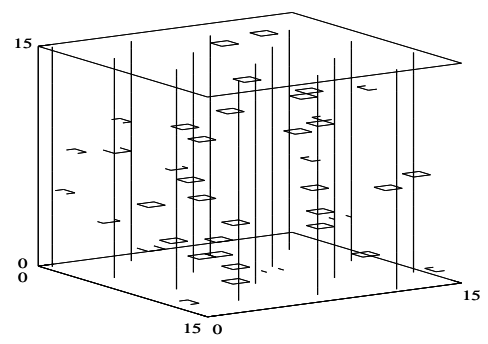

FIG. 9. Snapshot of the vortex-line configuration in the liquid phase for $R=0.04, B=1 / 15, \lambda_{1}=5, L_{x}=L_{y}=$ $L_{z}=15, T=0.25$.
Summarizing the above results, we find that $\mu_{y}$ generally tends to rise at significantly lower temperatures than $1-\gamma_{z}$. We will now present a consistency check on these response coefficients. We do this by considering the $B=0$ superconductor-to-normal transition that is driven by (the unbinding of) thermally excited vortex loops. In this transition, the loss of superconducting coherence is accompanied by a peak in the specific heat and a sharp increase in the number of vortex loops [26], and the results for the response coefficients should therefore match these features.

In Fig. 6 we present the results for $\mu_{y}$ following Carneiro together with the helicity modulus $\gamma_{z}$ following Chen and Teitel, calculated in the same simulation for an isotropic system at $B=0$. We find that $\mu_{y}$ rises at much lower temperatures than $1-\gamma_{z}$. The peak in the specific heat, an independent signature of the transition from the superconducting to the normal state, is situated around $T \approx 5.9$ and coincides with the rise in $1-\gamma_{z}$. This provides strong evidence that $\gamma_{z}$ and not $\mu_{y}$ is the correct quantity to measure the superconducting coherence. In Fig. 6 we also show the absolute vorticity per unit cell as a function of temperature, which is a measure of the number of thermally excited vortex loops. The increase in the vorticity is strongest close to $T \approx 5.9$, which is another signature of the transition [27,15]. The rise in $\mu_{y}$ takes place at temperatures where the number of vortex-loop fluctuations is still very small and where superconductivity can therefore not yet be destroyed. As insets in Fig. 6 we show snapshots of the vortex loop configurations for $T=4$ and $T=5.7$. We conclude that fluctuations in the net vorticity $q_{y}$ that are induced by the allowed incomplete loops in the planes $x=1$ and $x=L_{x}$ and that lead to a nonzero $\mu_{y}$, do not signal a loss of superconducting coherence in the bulk [28].

\section{B. Anisotropic case.}

We now discuss results for a moderately anisotropic case in a non-zero applied field. In Figs. 7 and 8 we compare results for $R=1 / 25$ (or $\Gamma=5$ ), corresponding to YBCO. Here we took $B=1 / 15$ and $\lambda_{1}=5$. The curve with data points shown as stars in Fig. 7 is the helicity modulus $\gamma_{z}$ following Chen and Teitel, or more precisely $\gamma_{z}\left(k_{x}\right)$ at the lowest $k_{x}$. The results for $1-\gamma_{z}\left(k_{x}\right)$ are shown in Fig. 11. The lattice size was taken to be $L_{x}=L_{y}=30, L_{z}=10$. We observe that for this anisotropic case, $1-\gamma_{z}$ rises to unity approximately at the vortex-lattice melting temperature. Similar results were obtained in a recent work by Nguyen et al. [16], where the effect of anisotropy was studied systematically. Due to the anisotropy, the energy cost of vortex-loop fluctuations parallel to the $x-y$ planes is reduced. As explained in Ref. [16], these parallel loops, which are most easily nucleated near a vortex line running in the $z$ direction, contribute to the decoupling of the planes, so that lon- 
gitudinal coherence is lost at lower temperature than for the isotropic case. In Ref. [16] it was shown in addition that for strong anisotropy $R \leq 1 / 100$, the parallel loops proliferate already at a temperature (far) below the melting temperature of the vortex lattice, thus giving rise to a vortex lattice phase without longitudinal coherence in between these temperatures.

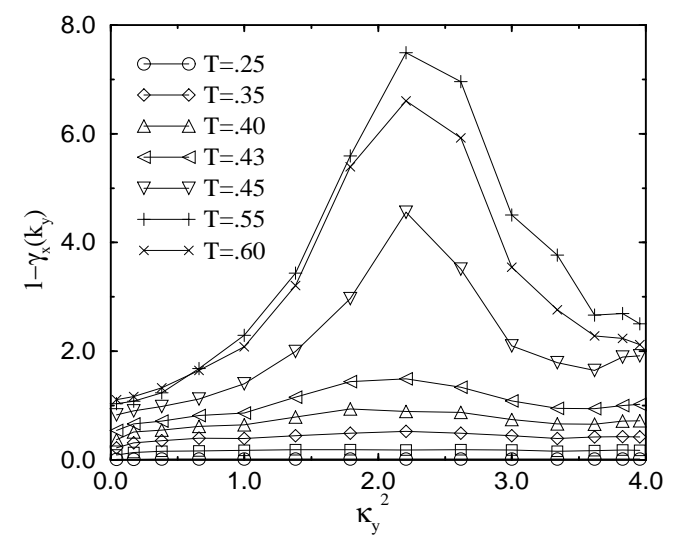

FIG. 10. Transverse response $1-\gamma_{x}\left(k_{y}\right)$ for different temperatures, calculated at constant $B$, for $R=0.04, B=1 / 15$, $\lambda=5, L_{x}=L_{y}=30, L_{z}=10$. The peak for higher $\mathrm{T}$ is at a wave vector commensurate with the vortex lattice.

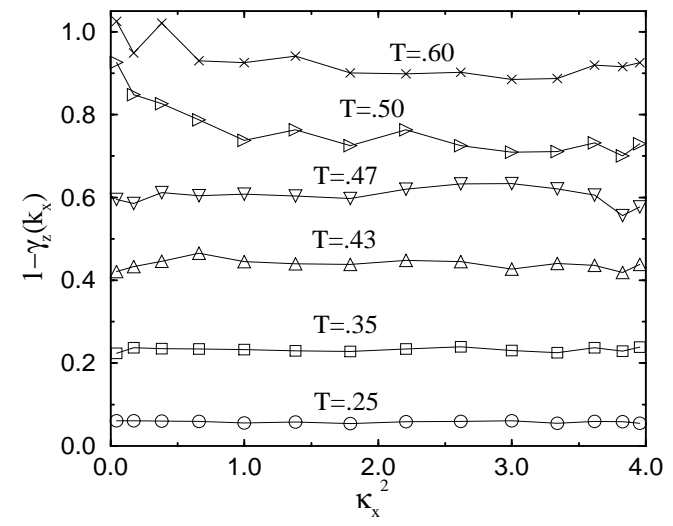

FIG. 11. Transverse response $1-\gamma_{z}\left(k_{x}\right)$ for different temperatures, calculated at constant $B$, for $R=0.04, B=1 / 15$, $\lambda=5, L_{x}=L_{y}=30, L_{z}=10$.

In Fig. 8 we show Carneiro's permeability $\mu_{y}$ calculated at the same parameters as considered in Fig. 7 . Here we used $L_{x}=L_{y}=L_{z}=15$. We observe that there is a similar reduction of the temperature at which longitudinal coherence is lost when compared to the isotropic case. However, $\mu_{y}$ rises already well below the melting temperature. The only physical mechanism that could be responsible for this early rise would be a proliferation of parallel loops. However, there is no such proliferation at this temperature, as can be seen from the snap- shot of the vorticity configuration at $T=0.25$ shown in Fig. 9. There are only few parallel loops present, as can be seen from the absolute vorticities shown in Fig. 8 as well. However, according to the result for $\mu_{y}$, the planes should be almost decoupled at this temperature. This again gives strong evidence that $\mu_{y}$ is governed by artefacts. In Fig. 8 we also show $1-\gamma_{z}$ calculated simultaneously from the fluctuations of $q_{x}$ at finite $k_{y}$. In agreement with the calculations shown in Fig. 7, this longitudinal response is found to turn resistive at the vortex-lattice melting temperature.

\section{SUMMARY}

We investigated the occurrence of longitudinal superconductivity in the vortex liquid phase in a clean type-II superconductor, within the lattice London model, motivated by recent contradicting results reported in Refs. [4] and 14]. For an isotropic superconductor, our results show three distinct regimes as a function of temperature. Between the Abrikosov lattice phase and a vortex liquid without longitudinal coherence, there is a liquid regime in which longitudinal superconducting coherence extends over length scales larger than the system thickness studied [4]. In the context of flux-transformer experiments, this intermediate regime should correspond to resistive regime with $z$-correlated vortex motion for samples that are sufficiently thin. In the moderately anisotropic case $R=1 / 25$ (corresponding to $\mathrm{YBCO}$ ), we find that longitudinal superconducting coherence is lost close to the flux-line lattice melting temperature. This is due to a proliferation, close to the melting temperature, of vortex loops between the layers [16]. In recent flux-transformer work [30] on YBCO single crystals, flux-line lattice melting and a loss of maximally $z$-correlated flux motion was found to coincide.

\section{ACKNOWLEDGMENTS}

We thank H.G. Evertz, M. Kraus, H. Nordborg and A. van Otterlo for discussions, and G. Carneiro as well as A.K. Nguyen and A. Sudbo for sending us their work prior to publication. We gratefully acknowledge financial support by the "Bayerischer Forschungsverbund Hochtemperatur-Supraleiter (FORSUPRA)".

* Present address: Institut für Theoretische Physik, Technische Universität Dresden, Mommsenstrasse 13, 01069 Dresden, Germany

** Present address: Siemens AG, Research Laboratories, Paul-Gossen-Strasse 100, 91052 Erlangen, Germany. 
[1] G. Blatter, M.V. Feigel'man, V.J. Geshkenbein, A.I. Larkin and V.M. Vinokur, Rev. Mod. Phys. 66, 1125 (1994).

[2] E.H. Brandt, Rep. Progr. Phys. 58, 1465 (1996).

[3] D.R. Nelson, Phys. Rev. Lett. 60, 1973 (1988); D.R. Nelson and H.S. Seung, Phys. Rev. B 39, 9153 (1989).

[4] T. Chen and S. Teitel, Phys. Rev. Lett. 74, 2792 (1995).

[5] M.V. Feigelman, V.B. Geshkenbein, and V.M. Vinokur, JETP Letters 52, 546 (1990). M.V. Feigelman, V.B. Geshkenbein, L.B. Ioffe, and A.I. Larkin, Phys. Rev. B 48, 16641 (1993). M.V. Feigelman and L.B. Ioffe, condmat/9403032

[6] E. Frey, D.R. Nelson, and D. Fisher, Phys. Rev. B 49, 9723 (1994);

[7] Y.H. Li and S. Teitel, Phys. Rev. B 47, 359 (1993); 49, 4136 (1994).

[8] Y.H. Li and S. Teitel, Phys. Rev. B 45, 5718 (1992).

[9] L. Xing and Z. Tesanovic, Phys. Rev. Lett. 65, 704 (1990).

[10] H. Ma and S.T. Chui, Phys. Rev. Lett. 682528 (1991).

[11] S. Ryu, S. Doniach, G. Deutscher and A. Kapitulnik, Phys. Rev. Lett. 68710 (1992).

[12] R. Sasik and D. Stroud, Phys. Rev. Lett. 722462 (1994).

[13] R. Sasik and D. Stroud, Phys. Rev. Lett. 75, 2582 (1995);

Phys. Rev. B 489938 (1993), 49, 16074, 50, 3294 (1994); R. Sasik, D. Stroud and Z. Tesanovic, Phys. Rev. B 52, 3042 (1995).

[14] G. Carneiro, Phys. Rev. Lett. 75, 521 (1995).

[15] G. Carneiro, Phys. Rev. B 53, 11837 (1996).

[16] A.K. Nguyen, A. Sudbo, and R.E. Hetzel, Phys. Rev. Lett. 77, 1592 (1996).

[17] T. Chen and S. Teitel, Phys. Rev. Lett. 76, 714 (1996).

[18] R. Cavalcanti, G. Carneiro and A. Gartner, Europhys. Lett. 17, 449 (1992); G. Carneiro, R. Cavalcanti and A.
Gartner, Phys. Rev. B 47, 5263 (1993).

[19] G. Carneiro, Phys. Rev. B 50, 6982 (1994).

[20] C. Dasgupta and B.I. Halperin, Phys. Rev. Lett. 47, 1556 (1981); S.E. Korshunov, Europh. Lett. 11, 757 (1990).

[21] T. Chen and S. Teitel, Phys. Rev. Lett. 72, 2085 (1994).

[22] E.H. Brandt, J. Low Temp. Phys. 44, 59 (1981).

[23] Thus the dominant $k_{\nu}$ dependence of the vorticity correlation $\left\langle q_{\sigma}\left(k_{\nu}\right) q_{\sigma}\left(-k_{\nu}\right)\right\rangle$ is described by the denominator in the second term in Eq. (6). From the functional form of this dependence one may indeed infer that an extrapolation to $k_{\nu}=0$ of the unnormalized correlation (i.e. the numerator in Eq. (6)) using a polynomial fit can only be successful when data points are available for $k_{\nu}$ values significantly smaller than $1 /\left(\lambda\left(1+\delta_{\mu, z}(\Gamma-1)\right)\right)$.

[24] In MC studies of a uniformly frustrated $X Y$ model on a cubic lattice, an intermediate disentangled vortex-liquid phase was found for a magnetic frustration $f=1 / 25$ [7], but not at $f=1 / 58$ for this reason.

[25] The decrease of $T_{m}$ with decreasing $B$ is due to the weakening of the artificial pinning.

[26] C. Dasgupta and B.I. Halperin, Phys. Rev. Lett. 47, 1556 (1981).

[27] G. Kohring, R.E. Shrock, and P. Wills, Phys. Rev. Lett. 57, 1358 (1986).

[28] We note that this may be less apparent from $B=0$ results for smaller values of $\lambda$, such as considered in $B=0$ calculations in Ref. 15.

[29] H. Safar, P.L. Gammel, D.A. Huse, S.N. Majumdar, L.F. Schneemeyer, D.J. Bishop, D. López, G. Nieva, and F. de la Cruz, Phys. Rev. Lett. 72, 1272 (1994); D. López, G. Nieva, and F. de la Cruz, Phys. Rev. B 50, 7219 (1994);

[30] D. López, E.F. Righi, G. Nieva, and F. de la Cruz, Phys. Rev. Lett. 76, 4034 (1996). 